\title{
A emergência do conceito saúde global: perspectivas para o campo da saúde coletiva
}

The emergency of concept global health: perspectives for the field of public health

La emergencia del concepto salud global: perspectivas para el campo de la salud pública

\author{
João Roberto Cavalcante Sampaio ${ }^{1}$ \\ Miriam Ventura ${ }^{2}$
}

\begin{abstract}
RESUMO: Nos últimos anos temos presenciado a emergência de novos termos no debate acadêmico e político da saúde coletiva, tais como: "saúde global", "bens públicos globais", "governança global em saúde", "saúde pública global", "diplomacia da saúde", "cooperação internacional". Este trabalho teve como objetivo analisar o desenvolvimento histórico do conceito "saúde global" e as perspectivas desta nova concepção na pesquisa e na prática da saúde coletiva. Foi realizada uma revisão bibliográfica nas bases BVS, Pubmed, Scielo e Scopus, identificados 514 trabalhos e selecionados 36 artigos científicos diretamente relacionados com 0 objeto de análise. Também foram selecionados documentos nos sites das organizações internacionais de saúde. Observou-se que a concepção de "saúde global" refere-se à saúde como um fenômeno transnacional ligado à globalização, que traz como principal desafio pensar a saúde pública para além das relações internacionais entre países. As organizações internacionais de saúde ganham especial importância no desenvolvimento da concepção de "saúde global", e suas novas perspectivas de aplicação no âmbito da saúde pública, são a diplomacia da saúde, a cooperação internacional e a governança da saúde global.
\end{abstract}

Palavras-chave: Saúde Global. Saúde Internacional. Saúde Pública Global. Saúde Mundial.

ABSTRACT: In recent years, we have witnessed the emergence of new terms in the academic and political debate of public health, such as "global health", "global public goods", "global health governance", "global public health", "health diplomacy", 'international cooperation". In this study, we aimed to analyze the historical development of the concept of 'global health', as well as the prospects of this new concept in the research and public health practice. A comprehensive literature review was performed in Pubmed, Scielo, Scopus, and BVS. We also analyzed documents obtained from the websites of international health organizations. 514 publications were retrieved and 36 were selected for this study. In general, the concept of "global health" refers to health as a transnational phenomenon linked to globalization, which has as main challenge to think public health beyond international relations between countries. International health organizations are particularly important in the development of the concept of "global health" and its new

\footnotetext{
${ }^{1}$ Bacharel em saúde coletiva, especialista em direito e saúde e saúde da família. Consultor internacional para a Organização Pan-Americana da Saúde para o Ministério da Saúde, Departamento de Vigilância das Doenças Transmissíveis. Brasília - Distrito Federal. Brasil. E-mail: joao.rcs@hotmail.com

2 Doutora em saúde pública. Professora adjunta do Instituto de Estudos em Saúde Coletiva da Universidade Federal do Rio de Janeiro. Rio de Janeiro - Rio de Janeiro. Brasil. E-mail: miriam.ventura@iesc.ufri.br
} 
application prospects in the field of public health are health diplomacy, international cooperation and global health governance.

Keywords: Global Health. International Health. Global Public Health. Worldwide Health.

RESUMEN: En los últimos años hemos presenciado la emergencia de nuevos términos en el debate académico y político de la salud colectiva, tales como: "Salud Global", "Bienes públicos globales", Gobernación Global en salud", "Salud pública global", "Diplomacia de la salud", "cooperación internacional". Este trabajo tuvo como objetivo analizar el desarrollo histórico del concepto "salud global" y las perspectivas de esta nueva concepción en el campo de investigación y en la práctica de Salud Colectiva. Fue realizada una revisión bibliográfica en bases BVS, Pubmed, Scielo y Scopus, identificado 514 trabajos y seleccionados 36 artículos científicos directamente relacionados con el objeto de análisis. También fueron seleccionados documentos en las páginas web de las Organizaciones internacionales de salud. Se observó que la concepción de "salud global" se refiere a la salud como un fenómeno transnacional ligado a la globalización, que trae como principal desafío pensar en la salud pública para más allá de las relaciones entre los países. Las organizaciones internacionales de la salud ganan especial importancia en el desarrollo de la concepción de "salud global", y sus nuevas perspectivas de aplicación en el ámbito de la salud pública, son la diplomacia de la salud, la cooperación internacional y la gobernación de la salud global.

Palabras-llaves: Salud Global. Salud internacional. Salud pública global. Salud mundial.

\section{Introdução}

O século XXI presencia a emergência de novas discussões e conceitos no âmbito da saúde coletiva, tais como "saúde global", "bens públicos globais", "governança global em saúde","saúde pública global", "diplomacia da saúde", "cooperação internacional" (1). Com frequência discursos oficiais de autoridades políticas, documentos de organizações internacionais de saúde e a literatura científica têm se referido a esses novos objetos de estudo (1), e suas repercussões no contexto acadêmico e de formação tornando-se a expressão saúde global dominante nas discussões .

Observa-se que o uso do termo "saúde global" foi sendo incorporado em substituição ao termo "saúde internacional"(criado em 1913 pela Fundação Rockfeller), para se referir à saúde como um fenômeno transnacional, que deve ser considerado para além das relações internacionais entre países. (1). Para Brown (2), os termos 'internacional', 'intergovernamental' e 'global' não são mutuamente excludentes e podem ser entendidos como complementares. A palavra 'global', para este autor, está associada à importância de inclusão de novos atores além das agências e organizações internacionais governamentais e intergovernamentais na discussão sobre saúde no âmbito internacional. 
Berlinguer (3) aponta que a ideia de saúde global emerge da necessidade de que cada país atue em um contexto amplo e que não se restrinja a fronteiras. A ideia de saúde global relaciona-se também às discussões sobre os efeitos da globalização na dinâmica do processo saúde/doença e cuidado, e as novas exigências e desafios que este fenômeno traz à saúde das populações. O mesmo autor aponta, ainda, que a globalização "pode ser resumida como a internacionalização da produção e do consumo, dos valores e costumes por meio do movimento de capital, força de trabalho, tecnologia e informação", [e] "a crescente mobilidade de pessoas, produtos e serviços, acelera a transferência de riscos de doenças".

Para Kickbusch (4) a comunidade científica tem discutido saúde global como uma questão de política externa. Essa perspectiva está presente na Declaração de Oslo "Saúde Global: uma Premente Questão de Política Externa do Nosso Tempo" que foi lançada em 2007 na reunião ocorrida durante a 64aㅗ Assembleia Mundial da Saúde pelos Ministérios das Relações Exteriores do Brasil, França, Indonésia, Noruega, Senegal, África do Sul e Tailândia, que buscou ampliar a consciência sobre os problemas de saúde nas arenas de discussões e decisões de política externa.

No Brasil, se identifica uma maior discussão sobre o tema da Saúde Global relacionado à Diplomacia da Saúde, e a criação de instâncias institucionais que trabalhem com o tema.

Esse novo campo veio se estruturando na última década e atualmente engloba a cooperação internacional em saúde, a diplomacia em saúde global e a governança em saúde global, dentro do âmbito da saúde coletiva (5).

A compreensão desta nova dinâmica e o desenvolvimento de políticas e ações de saúde pública tem exigido um intenso diálogo entre acadêmicos, gestores e outros profissionais de saúde.

A Organização Mundial da Saúde (OMS) e a Organização Pan-americana da Saúde (OPAS) são agências intergovernamentais que desempenham funções internacionais de destaque no contexto da saúde global. Além dessas agências, enfatiza Brown (2), temos a presença efetiva de novos atores, como a mídia e organizações não governamentais e fundações, cujo trabalho tem tido alcance global e podem provocar mudanças significativas na vida e saúde das populações. 
Considerando a emergência do conceito de saúde global, a pesquisa empreendida teve como objetivo responder a seguinte questão: Quais as novas perspectivas trazidas pela saúde global para a pesquisa e prática na saúde coletiva?

\section{Métodos}

Foi realizada uma revisão da literatura nas principais bases virtuais de periódicos científicos relacionados ao tema - Biblioteca Virtual em Saúde (BVS), SciELO, Pubmed e Scopus, até setembro de 2013, disponíveis nessas bases.

Os termos de busca utilizados foram "saúde global", "saúde internacional" e "saúde pública global". Buscando-se o refinamento da pesquisa, foi pesquisado descritores identificados na lista dos Descritores de Ciências da Saúde (DeCS) "saúde mundial", "revisão" e "história"associados aos termos de busca. O operador booleano "AND" foi utilizado para a interseção dos descritores e palavras-chave. Os resumos dos artigos identificados foram lidos e selecionados os que atendiam o objeto da pesquisa para análise. Os critérios de inclusão considerados foram: artigos de revisão e pesquisa no idioma português, inglês e espanhol, disponíveis na internet, que abordassem o desenvolvimento histórico do conceito "saúde global" e sua aplicação no campo prático e de pesquisa da saúde coletiva.

Ao todo foram identificados 514 resumos excluídos os repetidos. Desse total, 32 artigos foram considerados adequados ao objeto da pesquisa para leitura completa dos textos.

Por fim, realizou-se a leitura exaustiva deste material à luz das principais referências teóricas da saúde coletiva, classificando os achados segundo as principais categorias que emergiam dos artigos sobre as questões da investigação.

Observou-se que até o ano 2000 o termo mais utilizado foi saúde internacional, e de 2000-2013 um crescimento significativo de artigos científicos e outros textos referindo-se a saúde global. A saúde global tem crescido como campo de pesquisa, o levantamento bibliográfico apontou que o número de artigos e textos sobre saúde global aumentou consideravelmente nos últimos anos, no campo da saúde coletiva. O resultado sugere um espécie de substituição da ideia de saúde internacional para a de saúde global, e a introdução de novas propostas para o enfrentamento dos desafios contemporâneos no mundo globalizado. Essas novas perspectivas serão apresentadas a seguir. 
Complementarmente foram consultados os sítios das Organizações das Nações Unidas (ONU), OMS e OPAS para busca de documentos sobre o tema. A discussão adiante apresentada será desenvolvida considerando-se esta categorização dos achados abordando-se a: "diplomacia e saúde", "cooperação internacional e saúde" e "governança da saúde global"temas que serão introduzidos com um breve histórico das perspectivas dos autores para a saúde global.

\section{Resultados}

\section{Diplomacia da Saúde Global}

Diplomacia é a arte e a prática da condução de negociações (4). Ela é compreendida como meio de conduzir as relações internacionais, mediante a intervenção de diplomatas. A prática diplomática pode ser dividida em dois tipos:

A primeira (bilateral) é uma diplomacia de tipo mais clássico, voltada principalmente para a condução das relações de um país para outro; e a diplomacia multilateral mostra mudanças nessas relações tradicionais (4).

Segundo o mesmo autor a diplomacia nos nossos dias precisam assegurar "bens públicos globais" independentemente de negociações na área de comércio, desenvolvimento econômico. A saúde das populações e as questões ambientais constituem bens públicos globais.

A 'Diplomacia da Saúde Global' visa compreender tais processos de negociações, níveis e atores múltiplos que moldam e dirigem o ambiente da política global da saúde. Nesse sentido, Kickbusch (4) aponta três objetivos da diplomacia da saúde global :

1) contribuir para garantir melhor segurança em saúde e nos efeitos sobre a saúde da população de todos os países envolvidos (desta maneira atendendo aos interesses nacionais e globais);

2) contribuir para melhorar as relações entre países e fortalece o compromisso de uma ampla faixa de atores no trabalho de melhorar a saúde;

3) oferecer a compreensão da saúde como resultado de um esforço comum para assegurar que ela seja um direito humano e um bem público global, com metas de resultados que sejam considerados justos 'para todos' (isto é redução da pobreza, aumento da igualdade). 
Segundo Buss (6) o conceito da 'diplomacia da saúde global' emergiu para tratar dos fatores da saúde que transcendem as fronteiras nacionais e expõe os países às influências globais. Portanto, para este autor, esse conceito também possibilita uma coordenação melhor entre os setores de saúde e relações exteriores dos governos.

A diplomacia da saúde vem sendo considerado um campo novo de conhecimento e prática cujo objeto é a saúde e as negociações internacionais em torno dela, para o qual convergem diferentes disciplinas e profissionais, como diplomatas e profissionais de saúde na formulação e desenvolvimento de ações e intervenções globais (1).

A OMS é a principal organização de atuação da diplomacia da saúde global, mas novos atores e cenários, como as ONG's e organizações sociais, se expandem com rapidez. A diplomacia da saúde global também está presente na Organização Mundial do Comércio (OMC), no Banco Mundial, nas organizações regionais e nas novas organizações como alianças, fundos e fóruns globais (7).

A diplomacia da saúde global serve de campo de prática não só para os profissionais das relações internacionais, mas também para os sanitaristas, entre outros profissionais.

\section{Cooperação Internacional em Saúde}

A cooperação internacional em saúde é uma prática antiga na história mundial que ampliou-se após as novas dinâmicas da globalização. Infelizmente, a prática da cooperação tem acentuado disparidades entre os países e acarretado tensões e conflitos sociais e de saúde, em especial nos países mais afastados do circuito central da economia global, como nos países pobres da África, por exemplo (8).

Para Buss (7), neste início de século $X X I$, as condições de saúde de grandes parcelas da população de diversos países do mundo vêm se deteriorando. A questão relativa a segurança alimentar e as consequências das mudanças climáticas para saúde, por exemplo, têm chamado atenção da comunidade internacional.

O progresso científico e políticas adequadas de saúde pública não tem alcançado muitos países da África e da América Latina nos quais há o convívio com doenças transmissíveis epidêmicas, emergentes, reemergentes e negligenciadas, como o HIV/AIDS, Malária e Tuberculose, assim como com doenças crônicas não-transmissíveis (DCNT), entre as quais doenças cardiovasculares, diabetes, obesidade, neoplasias e doenças mentais (9). Além disso, pobreza, fome, subnutrição e atenção insatisfatória à 
maternidade, infância e idosos são responsáveis pelas elevadas mortalidades em geral, materna e de menores de 5 anos, bem como a uma baixa expectativa de vida ao nascer. 0 perfil epidemiológico dessas regiões também apontam iniquidades nas condições sociais e de saúde, em especial o acesso aos serviços de saúde existentes, públicos ou privados (9). Os profissionais de saúde disponíveis são escassos nos países mais pobres e em desenvolvimento, e em geral poucos são capacitados e a maioria com baixa remuneração (7).

Em relação às fragilidades dos sistemas de saúde desses países, Buss (7) afirma que "[a] maioria dos países pobres são incapazes de enfrentar as necessidades de suas populações, as doenças prevalentes, seus principais fatores de risco e as péssimas condições de vida que afetam a saúde, o que os coloca em situação de grande dependência da ajuda internacional, crucial tanto para o desenvolvimento quanto para a melhoria das condições de vida e saúde de suas populações" (7).

Nesse sentido, a ONU, as agências de cooperação dos países mais desenvolvidos do mundo e a filantropia internacional vem buscando responder essas grandes preocupações incluindo a saúde como prioridade na agenda da cooperação internacional e dos programas de ajuda para o desenvolvimento (9). Mas, as fragilidades do próprio sistema da OMS no sentido de atuar de forma mais efetiva na redução da iniquidades em saúde e controle dos vetores de doença têm sido apontadas por pesquisadores e a própria Direção-Geral da organização em prol de uma reforma (10).

Segundo Buss `A saúde global e a diplomacia da saúde exigem novos mecanismos institucionais e profissionais de saúde pública, levando a que diversas instituições acadêmicas do mundo estabeleçam centros de pesquisa e formação de recursos humanos dedicados ao tema" (11). Observa que no plano internacional tem se organizado alianças

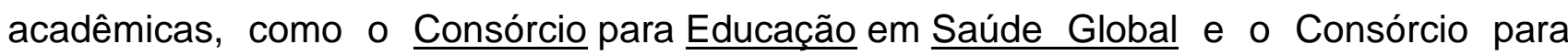
Diplomacia em Saúde Global. Porém, para este autor, ainda existe uma forte competição entre as nações, e somente quando a cooperação, amizade e solidariedade internacional superarem tal rivalidade, é que as nações vão conseguir atingir de fato o íntimo da saúde global.

De acordo Keusck (12), a ONU, OMS, Fundo das Nações Unidas para a Infância (Unicef), Programa das Nações Unidas para o Desenvolvimento (PNUD) e outras, vem 
incentivando os países a assinarem termos de cooperação internacional, por exemplo, para o cumprimento dos Objetivos do Milênio (ODM).

Sobre a questão, observa Buss que mesmo munidos das melhores intenções (ajudar as populações dos países pobres), os países que se beneficiam da cooperação muitas vezes não conseguem organizar a própria demanda e acabam por não terem resultados satisfatórios no que se diz respeito à distribuição dos recursos destinados (8).

Keusch (12) avaliou os efeitos das parcerias globais no campo da saúde em vinte países em desenvolvimento e chegou à seguinte conclusão:

\begin{abstract}
Infelizmente, os ganhos obtidos pelas Parcerias em Saúde Global tiveram um custo. A introdução 'vertical' de recursos em sistemas de saúde organizados horizontalmente, em um ambiente com recursos restritos, tende a criar diversas consequências para os países beneficiários' (12)
\end{abstract}

Os diversos atores da saúde global passaram buscar alternativas aos modelos dominantes dos países do hemisfério norte, eis que surgiu a cooperação sul-sul, que pode ser definida como o "processo de interação econômica, comercial, social ou de outra natureza que se estabelece em desenvolvimento, geralmente localizados no hemisfério sul". (7).

A cooperação Sul-Sul nas áreas sociais vem recebendo crescente atenção dos países em desenvolvimento do hemisfério sul, seja pelas limitações relativo da cooperação tradicional Norte-Sul, dominada pela visão e práticas centralizadoras dos países desenvolvidos, seja pela entrada de países emergentes de renda média no cenário da cooperação internacional, seja, ainda, pelos benefícios que países mais pobres vislumbram nesta 'cooperação entre semelhantes. (13)

O Brasil por exemplo, vem firmando acordos de cooperação com os países da África e alguns países da América Latina. A cooperação internacional em saúde é mais um objeto de pesquisa e prática para a saúde coletiva. (13)

\title{
Governança da Saúde Global
}

Um tema que tem rondado o panorama da saúde global e que sofreu consideráveis alterações nos últimos anos foi o de Governança da Saúde Global. Para Kickbusch (4), o número de organizações que se ocupam de questões de saúde apresentou um aumento 
exponencial. O crescimento de parcerias público-privadas, de doadores, fundos e outros atores, contribuíram para a diversificação dos envolvimentos no campo da saúde global.

A governança clássica da saúde internacional estruturava-se na crença de que os governos tinham a responsabilidade primária pela saúde de suas populações e portanto estavam aptos a realizar cooperação com outros países, a fim de proteger sua população dos riscos à saúde. A competência técnica exclusiva dos governos para atuar no âmbito da cooperação internacional tem sido relativizada incluindo-se atores não governamentais na governança da saúde global, e o trabalho humanitário e do setor privado para atender aspectos dessa cooperação. (4).

Segundo Krasner (1983) a discussão sobre governança em saúde global tem demandado investimento na criação, conformação, orientação, fortalecimento das instituições internacionais e de um arcabouço de princípios, normas, regras e procedimentos para tomadas de decisões tanto no âmbito organizacional como na promoção e proteção da saúde em escala global (14).

\section{Considerações finais}

A saúde global traz a ideia de que a saúde das pessoas do mundo deve ser sustentada por um esforço coletivo internacional, sem desprezar-se as especificidades locais. Deve-se, portanto, buscar compreender os fenômenos locais em articulação com as questões globais. O objetivo principal da ideia de saúde global é o de superar as dificuldades mundiais de saúde de forma coletiva e cooperativa, sem fronteiras.

Em relação as políticas de saúde globais, evidenciou-se no estudo que existe uma demanda de reorganização dos países no que concerne a governança em saúde, que responda de forma satisfatória aos novos desafios relacionados à efetivação dos direitos humanos na saúde, ultrapassando o simples combate de epidemias e pandemias para uma assistência à saúde integral para a produção de bem estar a todos os cidadãos das diversas nações.

Observou-se, ainda, a relevância e o crescimento da atuação das organizações sociais internacionais de saúde, que realizam ações de saúde sustentáveis no apoio em países pobres, e no incentivo e promoção da cooperação internacional em saúde.

Um das propostas para a saúde global tem sido uma concepção favorável para a resposta à novas epidemias internacionais, tanto em países ricos, como em países pobres, 
mas para 0 alcance desses objetivos exige profissionais que possam compreender de forma mais ampla o processo saúde/doença e cuidado, e sejam capazes de formular e implementar ações que reduzam desigualdades e vulnerabilidades, locais e globais, que importam no adoecimento e deteriora a qualidade de vida de grandes segmentos populacionais.

\section{Referências}

1 Almeida C. A experiência da Fiocruz na formação de profissionais em saúde global e diplomacia da saúde: base conceitual, estrutura curricular e primeiros resultados. RECIIS: Revista Eletrônica de Comunicação, Informação \& Inovação em Saúde, v. 4, n. 1, p. 148164, mar. 2010. Disponível em: http://www6.ensp.fiocruz.br/repositorio/sites/default/files/arquivos/355-1619-1-PB.pdf>. Acesso em: 11 nov 2013

2 Brown M, Cueto M, Fee A. A transição de saúde pública 'internacional' para 'global' e a Organização Mundial da Saúde. História, Ciências, Saúde-Manguinhos, v. 13, n. 3, p. 623647, jul./set. 2006. Disponível em: <http://www.scielo.br/pdf/hcsm/v13n3/04.pdf>. Acesso em: 12 nov 2013

3 Berlinguer G. Globalização e saúde global. Estudos Avançados, v. 13, n. 35, abr. 1999. Disponível em: <http://www.scielo.br/pdf/ea/v13n35/v13n35a03.pdf>. Acesso em: 12 nov 2013

4 Kickbusch I. Diplomacia da Saúde Global. RECIIS: Revista Eletrônica de Comunicação, Informação \& Inovação em Saúde, v. 4, n. 1, p. 19-24, mar. 2010. Disponível em: $<$ http://www.reciis.icict.fiocruz.br/index.php/reciis/article/download/342/494>. Acesso em: 16 nov 2013

5 Buss $P$, Matida A. Declaração de Istambul sobre saúde global. Cadernos de Saúde Pública, v. 25, n. 9, p. 2083-2085, set. 2009. Disponível em: <http://www.scielo.br/pdf/csp/v25n9/22.pdf>. Acesso em: 13 nov 2013

6 Buss P. A UNASUL saúde. Le Monde Diplomatique Brasil, v. 26, n.30-31, set. 2008. <http://www.diplomatique.org.br/artigo.php?id=557>. Acesso em: 14 nov 2013.

7 Buss P, Ferreira JR. Ensaio crítico sobre a cooperação internacional em saúde. RECIIS: Revista Eletrônica de Comunicação, Informação \& Inovação em Saúde, v. 4, n. 1, p. 93105, mar. 2010. Disponível em:

$<$ http://pascal.iseg.utl.pt/ cesa/files/Comunicacoes/RPereira2.pdf>. Acesso em: 13 nov 2013

8 Buss P. Globalização, pobreza e saúde. Ciência \& Saúde Coletiva, v. 12, n. 6, p. 1575- 
1589, 2007. Disponível em: <http://www.scielo.br/pdf/csc/v12n6/v12n6a17.pdf>. Acesso em: 14 nov 2013.

9 ONU. Novo relatório da OMS traz informações sobre estatísticas de saúde em todo o mundo. maio 2011. Disponível em: <http://www.onu.org.br/novo-relatorio-da-oms-trazinformacoes-sobre-estatisticas-de-saude-em-todo-o-mundo/>. Acesso em: 17 nov 2013

10 Ventura D, Perez FA. Crise e reforma da organização mundial da saúde. Lua Nova, São Paulo, n. 92, p. 45-77, Aug. 2014. Disponível em: <http://www.scielo.br/scielo.php?script=sci_arttext\&pid=S010264452014000200003\&lng=en\&nrm=iso>. Acesso em: 14 nov 2013.

11 Buss P, Leal MC. Cad. Saúde Pública, Rio de Janeiro, 25(12):2540-2541, dez, 2009. Disponível em: http://www.scielosp.org/pdf/csp/v25n12/01.pdf. Acesso em 14 nov 2013

12 Keusch G, Wen L. The global health system: linking knowledge with action: learning from malaria. Plos Medicine, 7 (1): e1000179, 2010. Disponível em:

<http://www.plosmedicine.org/article/info\%3Adoi\%2F10.1371\%2Fjournal.pmed.1000179Ac esso em: 15 nov 2013

13 Buss P, Ferreira JR. Diplomacia da saúde e cooperação Sul-Sul: as experiências da Unasul saúde e do Plano Estratégico de Cooperação em Saúde da Comunidade de Países de Língua Portuguesa (CPLP). R. Eletr. de Com. Inf. Inov. Saúde. Rio de Janeiro, v.4, n.1, p.106-118, mar., 2010. Disponível em:

http://www6.ensp.fiocruz.br/radis/sites/default/files/pdf/diplomacia-da-saude-e-cooperacaosul-sul-as-experiencias-da-unasul-saude-e-do-plano-estrategico-de-cooperacao-em-saudeda-comunidade-de-paises-de-lingua-portuguesa-cplp.pdf . Acesso em 15 nov 2013

14 Krasner, S. International regimes, New York: Cornell University Press. 1983. Acesso em 16 nov 2013. Disponível em:

Recebido em: 28/7/2016 Aprovado em: 6/12/2016

\section{Como citar este artigo:}

Sampaio JRC, Ventura M. A emergência do conceito saúde global: perspectivas para o campo da saúde coletiva. Revista Cadernos Ibero-Americanos de Direito Sanitário. 2016 out./dez, 5(4):145-155. 